\title{
Digital Post-Processing Based Wideband Receiver Linearization for Enhanced Spectrum Sensing and Access
}

\author{
Markus Allén, Jaakko Marttila, and Mikko Valkama \\ Department of Electronics and Communications Engineering \\ Tampere University of Technology \\ P.O. Box 692, FI-33101 Tampere, Finland \\ \{markus.allen, jaakko.marttila, mikko.e.valkama\}@tut.fi
}

\author{
Michael Grimm and Reiner Thomä \\ Electronic Measurement Research Lab \\ Ilmenau University of Technology \\ 98684 Ilmenau, Germany \\ \{michael.grimm, reiner.thomae\} @tu-ilmenau.de
}

\begin{abstract}
Wideband radio receivers provide the flexibility desired in many communications applications and are the key element in cognitive radios and software-defined radios in general. However, multi-channel reception scenarios tend to have high dynamic range which set hard-to-reach requirements for receiver linearity. This paper proposes a calibration-based digital postinverse model for wideband receiver linearization and compares it with adaptive interference cancellation. Their advantages and disadvantages are highlighted together with numerical performance results in challenging non-contiguous spectrum access scenario. Both methods are waveform-independent, which make them applicable to many systems, but they have different tradeoffs when linearization accuracy, computational complexity and real-time capability are compared. Therefore selecting the best method is highly system-specific matter.
\end{abstract}

Index Terms-Interference cancellation, inverse modeling, nonlinear distortion, spectrum access, spectrum sensing

\section{INTRODUCTION}

Wideband radio receivers and especially the ones employing direct-conversion architecture have gained more and more attention during the last decade. This is due to their flexibility, cost efficiency, and integrability [1], [2]. Wideband receivers are attractive in many emerging software-defined radio and cognitive radio applications as well as in traditional cellular communications networks. However, wideband multi-channel reception scenarios set stringent receiver linearity requirements and these cannot be always met even with state-of-the-art receiver hardware [3], [4].

In practice, the linearity problems have been encountered, e.g., in field measurements with mobile spectrum sensing devices [5], [6]. Due to the limited dynamic range of the receiver, significant amount of nonlinear distortion is caused to vacant channels or on top of weak signals, if there are strong neighboring signals present at the same time. This deteriorates

This work was supported by the Finnish Funding Agency for Technology and Innovation (Tekes) under the project "Enabling Methods for Dynamic Spectrum Access and Cognitive Radio" within TRIAL technology programme, The Academy of Finland under the project 251138 "Digitally-Enhanced RF for Cognitive Radio Devices", Austrian Competence Center in Mechatronics (ACCM), and Tampere University of Technology Graduate School. spectrum sensing reliability in case of energy detection as well as when more advanced techniques, such as feature detectors, are utilized.

In some systems, the spectrum sensing problems can be avoided using a centralized database for providing information about vacant channels [7], [8]. However, spectrum access itself may be challenging due to the receiver nonlinearities. Especially non-contiguous spectrum access is challenging because there might be strong blocking signals between the desired channels causing nonlinear distortion [3], [4].

In mobile cellular radio systems, limited linearity of mobile devices is evident from their restrictions in size and cost. However, receiver nonlinearity challenges exist also in uplink communications. It is desired that wideband multi-standard base-stations are able to concurrently receive weak and strong signals with a single receiver chain. In addition, there might be strong blocking signals from co-located or nearby transmitters. For example, GSM, UMTS, and LTE specifications define blocker test scenarios which require at least $70 \mathrm{~dB}$ spuriousfree dynamic range [9]-[11]. In reality, worst case scenarios can be even more challenging.

As shown by the aforementioned use cases, the nonlinearities of wideband receivers are crucial to be considered in order to create practical, more flexible and commercially attractive communications systems, especially when cognitive radio paradigm is exploited. The most prominent approach to tackle the nonlinearity problem is the use of digital postprocessing. With behavioral modeling, versatile algorithms can be developed, which are applicable to many different kinds of systems. As one promising approach for receiver linearization, this paper proposes calibration-based post-inverse method. The principle itself is well known in the current literature [12]-[14], but a practical structure for receiver nonlinearity inverse model is an important aspect of this paper. Essential contribution is also comparison with another potential method called adaptive interference cancellation (AIC) [6], [15]-[17]. This is important since the methods have much in common, but have also some fundamental differences which may prevent or make possible their usage on certain applications.

The remainder of the paper is organized as follows. Sec- 


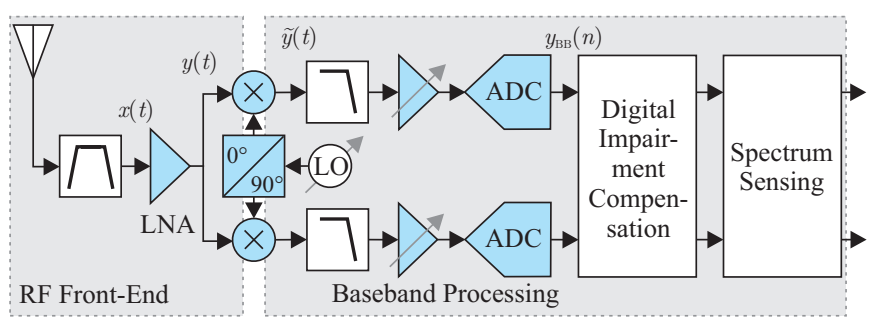

Fig. 1. Conceptual direct-conversion receiver block diagram highlighting the components which are considered as sources of nonlinearity and I/Q imbalance in this paper.

tion II presents mathematical modeling of receiver nonlinearities and discusses their effect on spectrum sensing and access. Section III introduces two nonlinearity compensation methods, namely AIC and calibration-based post-inverse. Performance simulation results and compensation method comparison are given in Section IV. Finally, the paper is concluded in Section $\mathrm{V}$.

\section{RECEIVER NONLINEARITY CHALLENGES IN SPECTRUM SENSING AND ACCESS}

This paper considers wideband direct-conversion receiver due to its integrability, cost-effectiveness, and general popularity. Fig. 1 illustrates a conceptual block diagram of a direct-conversion receiver. Components which are essential sources of nonlinearity and/or I/Q imbalance are highlighted. $\mathrm{RF}$, mixing, and baseband (BB) stages are cascaded in reality and therefore proper nonlinearity modeling requires taking into account their joint effect.

The nonlinearity modeling discussed in this section follows the concept described in [15]. Starting point is the received bandpass signal at the input of the low-noise amplifier (LNA), which has not been distorted by the receiver yet. Its BB equivalent version is

$$
x(t)=A(t) e^{j \phi(t)},
$$

which consist of the envelope $A(t)$ and phase $\phi(t)$. Please notice that in this paper $x(t)$ represents the overall received signal which may consist of several individual waveforms at different complex intermediate frequencies (IFs).

The RF distortion caused by the LNA is modeled here with a third-order polynomial. The essential part of the RF distortion can be modeled as

$$
y(t)=a_{1} x(t)+3 a_{2} A^{2}(t) x(t),
$$

where $a_{1}$ and $a_{2}$ are complex coefficients describing the nonideal LNA behavior and $y(t)$ is the BB equivalent LNA output. This models the intermodulation distortion (IMD) around the original carrier. Third-order RF nonlinearity also causes harmonics, but those are typically far away from the original carrier and since straightforward to filter out.

In I/Q down-conversion stage, the mixer causes some I/Q imbalance. It is classically modeled as follows:

$$
\tilde{y}(t)=k_{1} y(t)+k_{2} y^{*}(t),
$$

TABLE I

All the Terms Generated by the Cascaded Nonlinearity Model

\begin{tabular}{lll}
\hline \hline Terms & $\begin{array}{l}\text { Conjugate } \\
\text { Terms }\end{array}$ & Interpretation \\
\hline \hline$x(t)$ & $x^{*}(t)$ & Original undistorted signal \\
\hline$A^{2}(t) x(t)$ & $A^{2}(t) x^{*}(t)$ & 3rd-order IMD \\
{$\left[x^{*}(t)\right]^{3}$} & $x^{3}(t)$ & 3rd-order harmonics \\
\hline$A^{4}(t) x(t)$ & $A^{4}(t) x^{*}(t)$ & 5th-order IMD \\
$A^{2}(t)\left[x^{*}(t)\right]^{3}$ & $A^{2}(t) x^{3}(t)$ & IMD of 3rd-order harmonics (5th order) \\
\hline$A^{6}(t) x(t)$ & $A^{6}(t) x^{*}(t)$ & 7th-order IMD \\
$A^{4}(t)\left[x^{*}(t)\right]^{3}$ & $A^{4}(t) x^{3}(t)$ & IMD of 3rd-order harmonics (7th order) \\
\hline$A^{8}(t) x(t)$ & $A^{8}(t) x^{*}(t)$ & 9th-order IMD \\
$A^{6}(t)\left[x^{*}(t)\right]^{3}$ & $A^{6}(t) x^{3}(t)$ & IMD of 3rd-order harmonics (9th order) \\
\hline \hline
\end{tabular}

where $k_{1}=\left(1+g_{\mathrm{m}} e^{-j \phi_{\mathrm{m}}}\right) / 2$ and $k_{2}=\left(1-g_{\mathrm{m}} e^{j \phi_{\mathrm{m}}}\right) / 2$, which are based on gain mismatch $g_{\mathrm{m}}$ and phase mismatch $\phi_{\mathrm{m}}$ (in rad). Perfect I/Q balance would be achieved with $g_{\mathrm{m}}=1$ and $\phi_{\mathrm{m}}=0$. The mixer I/Q imbalance brings on mirror images of both the original signal $x(t)$ and its RF distortion.

After the mixer, the following analog stages are separate for the I and Q branches of the signal. This may cause some additional I/Q imbalance. In addition, the mixer and BB stages cause nonlinear distortion. Their effects are considered here together with a single model. Signal $\tilde{y}(t)$ after the thirdorder $\mathrm{BB}$ nonlinearity is denoted with $y_{\mathrm{BB}}(t)=B(t) e^{j \theta(t)}=$ $y_{\mathrm{I}, \mathrm{BB}}(t)+j y_{\mathrm{Q}, \mathrm{BB}}(t)$ and can be defined as

$$
\begin{gathered}
y_{\mathrm{I}, \mathrm{BB}}(t)=a_{3 \mathrm{I}} \tilde{y}_{\mathrm{I}}(t)+a_{4 \mathrm{I}} \tilde{y}_{\mathrm{I}}^{3}(t), \\
y_{\mathrm{Q}, \mathrm{BB}}(t)=a_{3 \mathrm{Q}} \tilde{y}_{\mathrm{Q}}(t)+a_{4 \mathrm{Q}} \tilde{y}_{\mathrm{Q}}^{3}(t),
\end{gathered}
$$

where real coefficients $a_{3 \mathrm{I}}, a_{3 \mathrm{Q}}, a_{4 \mathrm{I}}$, and $a_{4 \mathrm{Q}}$ describe the nonlinear behavior independently for I and Q branches. This leads to the overall cascaded nonlinearity model comprising third-order RF and BB nonlinearities as well as the essential I/Q imbalance behavior.

The signal after all aforementioned cascaded impairments, $y_{\mathrm{BB}}(t)$, can also be written using the undistorted signal $x(t)$. The equation consist of 18 different terms and for the sake of presentation clarity, the terms are tabulated in Table I. The first column lists the terms generated by the cascaded thirdorder RF and BB nonlinearities without any I/Q imbalance. The second column contains the terms generated if either mixer or BB I/Q imbalance or both occur. All the terms have a different weighting factor, which is a combination of $a_{1}$, $a_{2}, a_{3 \mathrm{I}}, a_{3 \mathrm{Q}}, a_{4 \mathrm{I}}, a_{4 \mathrm{Q}}, k_{1}$, and $k_{2}$. However, these weighting factors are omitted from Table I in order to keep it concise. Further details can be found from [15]. Typically, many of these 18 terms are buried under the noise floor and do not have to be considered in practice. However, it is easy to conceptually interpret the meanings of all the terms. They can be divided in two categories: IMD around the original IF and harmonics/IMD around triple the original IF (thirdorder harmonics zone). The exponent of the envelope $A(t)$ is proportional to the bandwidth of the distortion. 


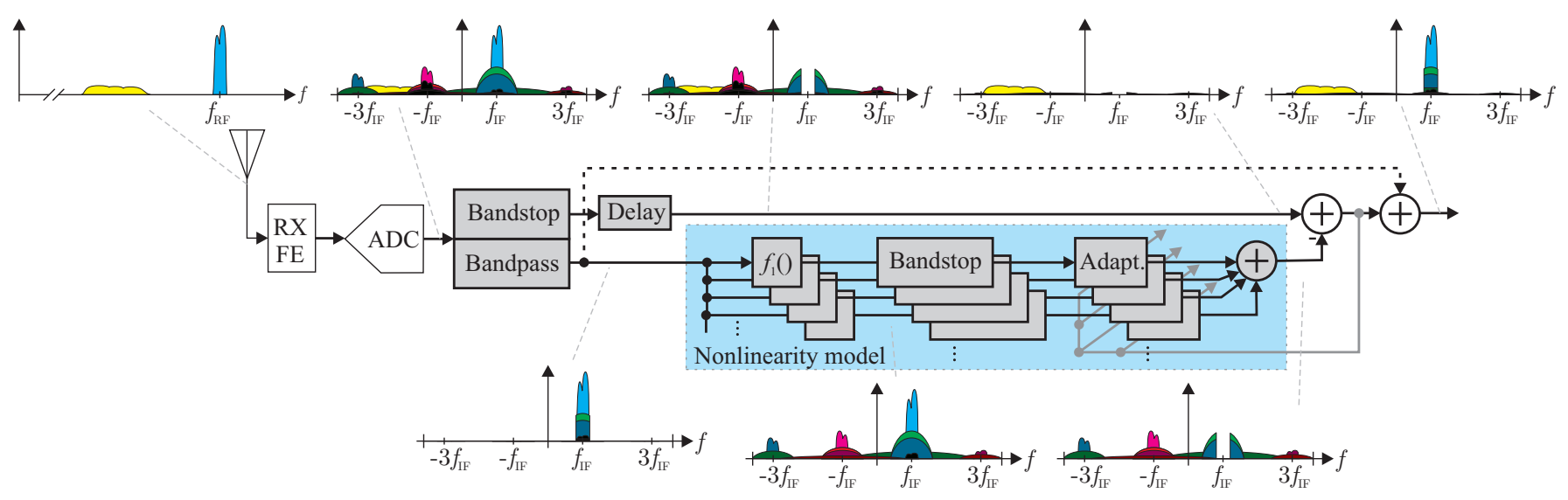

Fig. 2. Block diagram illustrating the principle of AIC method for receiver linearization. Conceptual spectra illustrate the processing flow for one weak signal and one strong blocking signal.

TABLE II

TERmS Used IN AIC FOR NONLINEARITY MODELING

\begin{tabular}{ll}
\hline \hline Terms & Interpretation \\
\hline \hline$\hat{x}^{*}(n)$ & Mirror image of the undistorted signal \\
$A^{2}(n) \hat{x}(n)$ & 3rd-order IMD \\
$A^{2}(n) \hat{x}^{*}(n)$ & Mirror image of 3rd-order IMD \\
{$\left[\hat{x}^{*}(n)\right]^{3}$} & 3rd-order harmonics \\
$\hat{x}^{3}(n)$ & Mirror image of 3rd-order harmonics \\
\hline \hline
\end{tabular}

The power of the distortion caused by receiver nonlinearities is proportional to the power of the signal. Therefore strong signals may cause significant amount of distortion to neighboring channels which can interfere with spectrum sensing. The interference impact is two-fold. On the one hand, the distortion may cause false alarms in energy detectors and also in cyclostationary feature detectors, if the distortion possess similar features as the original signal [6]. On the other hand, the distortion may mask weak signals so that feature detectors are not able to discover them, i.e., missed detections are caused.

Receiver nonlinearities may cause problems even if the spectrum sensing is not necessary. Vacant channels might be known, e.g., due to a priori information from a centralized database. However, spectrum access is challenging due to the receiver nonlinearities, if strong adjacent-channel signals are concurrently present. This is especially true in case of noncontiguous spectrum access, if the strong blocking signals are located between the exploited non-contiguous spectrum chunks.

\section{COMPENSATION OF ReCEIVER NONLINEARITIES}

This section gives descriptions of two feasible methods for receiver linearization. First method adaptively finds a receiver nonlinearity model for cancelling nonlinear distortion, where as second method uses calibration signal to find post-inverse model for receiver nonlinearities.

\section{A. Adaptive Interference Cancellation}

AIC principle is described in [15] as it is also employed in this paper. The basic idea is illustrated with a block diagram in Fig. 2. A digitized signal is split into main branch and reference branch. The latter one contains only the blockers whereas the main branch all the other received signal content except the blockers. The nonlinear distortion generated by the received front-end is re-generated in the reference branch by applying in parallel different polynomial terms to the blockers. In order to avoid over-complicated system, only the essential terms are used from the overall model described in Section II. The selected nonlinearity terms for the AIC are listed in Table II. Notation $\hat{x}(t)$ refers to the estimate of $x(t)$ which is obtained with the bandpass filter shown in Fig. 2. The re-generated nonlinear distortion is then subtracted from the main branch thus compensating the nonlinear distortion in the received signal. Proper adaptive weights (or filters in case of nonlinearities with memory) can be found by using, e.g., the classical least-mean square (LMS) algorithm. If blocker signals are also desired to be received, they can be added back to the main branch signal after the processing as suggested by the dashed line in Fig. 2.

\section{B. Calibration-Based Post-Inverse}

In general, the effect of polynomial nonlinearity can be compensated with a post-inverse model. However, finding an exact inverse can be challenging and typically even simple polynomials have inverses of infinite order [13], [14]. For compensating receiver nonlinearities, this paper proposes using a post-inverse having terms listed in Table III. These terms are based on receiver modeling described in Section II. Only the most essential terms are selected to limit the complexity. Compared to the AIC terms in Table II, inverse modeling requires also the linear signal term and higher order IMD term. The principle of the proposed post-inverse processing is shown in Fig. 3. During the normal receiver operation, the received distorted signal after ADC is fed to the post-inverse nonlinearity model which then outputs the less distorted signal. The weights (or filters in case of nonlinearities with memory) 


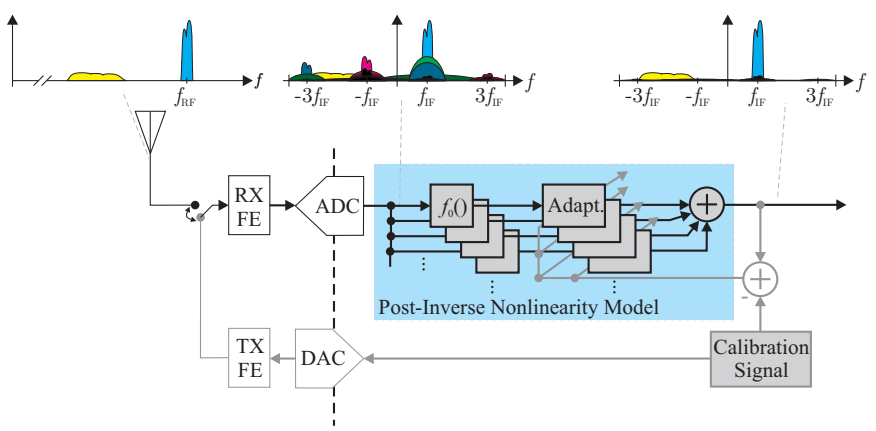

Fig. 3. Block diagram illustrating the principle of calibration-based postinverse for receiver linearization. Conceptual spectra illustrate the processing flow for one weak signal and one strong blocking signal.

TABLE III

TERMS USED IN INVERSE NONLINEARITY MODELING

\begin{tabular}{ll}
\hline \hline Terms & Interpretation \\
\hline \hline$y_{\mathrm{BB}}(n)$ & Distorted signal \\
$y_{\mathrm{BB}}^{*}(n)$ & Mirror image of distorted signal \\
$B^{2}(n) y_{\mathrm{BB}}(n)$ & 3rd-order IMD \\
$B^{2}(n) y_{\mathrm{BB}}^{*}(n)$ & Mirror image of 3rd-order IMD \\
{$\left[y_{\mathrm{BB}}^{*}(n)\right]^{3}$} & 3rd-order harmonics \\
$y_{\mathrm{BB}}^{3}(n)$ & Mirror image of 3rd-order harmonics \\
$B^{4}(n) y_{\mathrm{BB}}(n)$ & 5th-order IMD \\
\hline \hline
\end{tabular}

of the post-inverse nonlinearity model are adapted using a calibration signal. This can be done during the idle periods in the reception. Using the transmitter, the calibration signal is fed to the receiver front-end. Due to the known calibration signal, the weights/filters of the post-inverse nonlinearity model can be found using, e.g., the LMS algorithm. In principle, the calibration signal can be any wideband signal that the transmitter can properly generate and the digital version of it is known by the receiver.

\section{Simulation Results for Compensation Method COMPARISON}

Simulations examples in this section focus on a wideband OFDM mobile receiver capable of non-contiguous spectrum access. All essential simulation parameters are given in Table IV. A two-tone calibration signal with $-27 \mathrm{dBm}$ average power at the receiver input is used by the inverse method in all the simulations presented here.

First example considers two $10-\mathrm{MHz}$ wide blocker signals at down-converted center frequencies of $10 \mathrm{MHz}$ and $30 \mathrm{MHz}$, average received signal power being $-33 \mathrm{dBm}$. Spectrum illustration is given in Fig. 4. The mirror images of the blockers can be seen at $-10 \mathrm{MHz}$ and $-30 \mathrm{MHz}$. The IMD of the blockers is widely spread around the spectrum. It consists of spreading around the center frequencies of the blockers and also IMD caused by interaction between the blockers. The latter IMD can be partially seen as a spreading around $-10 \mathrm{MHz}$. In the simulations, the weights for the nonlinearity models in both
TABLE IV

SimULATION PARAMETERS

\begin{tabular}{lrl}
\hline \hline Parameter & \multicolumn{2}{c}{ Value } \\
\hline \hline LNA gain & $15 \mathrm{~dB}$ \\
LNA IIP3 & $-10 \mathrm{dBm}$ \\
Mixer IRR & $30 \mathrm{~dB}$ \\
Baseband gain & $35 \mathrm{~dB}$ \\
Baseband IIP3 & $5 \mathrm{dBm}$ \\
\hline Sampling rate & $80 \mathrm{MHz}$ \\
Quantization & $12 \mathrm{bits}$ \\
\hline Channel bandwidth & $10 \mathrm{MHz}$ \\
OFDM subcarrier spacing & $15 \mathrm{kHz}$ \\
Number of active subcarriers & 600 & \\
Subcarrier modulation & $16-\mathrm{QAM}$ & \\
Guard interval & $1 / 4$ & \\
\hline \hline
\end{tabular}

compensation methods are found with block least-squares in order to make the results as well comparable as possible. Fig. 4 illustrates also how well the compensation methods are able to remove the distortion. The post-inverse method performs slightly better due to the usage of calibration signal. AIC reference signal suffers from inband distortion of the blockers, because the reference is extracted from the received distorted signal. In addition, reference signal extraction causes degraded compensation performance at blocker band edges due to the bandpass filter transition bands.

Second example has two weak signals at center frequencies $-20 \mathrm{MHz}$ and $0 \mathrm{MHz}$ in addition to the blockers of the previous example. This scenario is shown in Fig. 5. It is evident from the spectrum that reception of the weak signals is impossible due to the vast amount of nonlinear distortion. However, both compensation methods are performing well enough so that the weak signals are clearly observable from the spectrum.

In order to provide wider view on the performance of the compensation methods, distortion rejection ratio (DRR) is used as a figure of merit. It is defined as

$$
D R R=\frac{S+N}{D},
$$

where $S, N$, and $D$ are signal, noise, and distortion powers, respectively. Fig. 6 presents wideband $D R R$ as a function of receiver signal power. Wideband DRR means that the values are calculated for the whole $80-\mathrm{MHz}$ reception band excluding blocker signal bands. Received signal power is stated as average power of the whole received waveform in the LNA input. Weak signals are always having the same $-80 \mathrm{dBm}$ average power per channel, but the power of the blockers is varied. It is also interesting look at DRR of a specific signal band. These narrowband $D R R$ values are given in Fig. 7 for the weak signal bands. When blocker power increases, the uncompensated narrowband DRR decreases and eventually there is more distortion power than useful signal power. The calibration-based post-inverse method is able to 


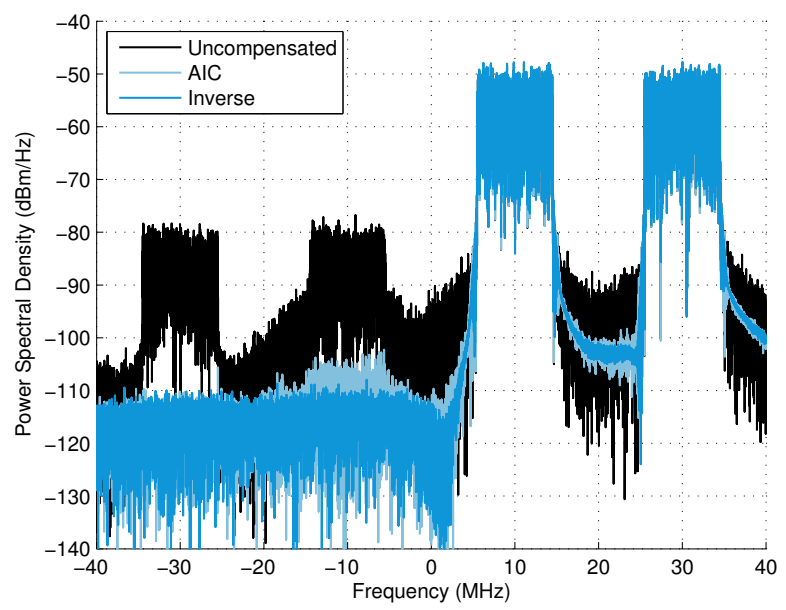

Fig. 4. Spectral illustration of nonlinearity compensation performance with two blocker signals having down-converted center frequencies $10 \mathrm{MHz}$ and $30 \mathrm{MHz}$, average received signal power being $-33 \mathrm{dBm}$.

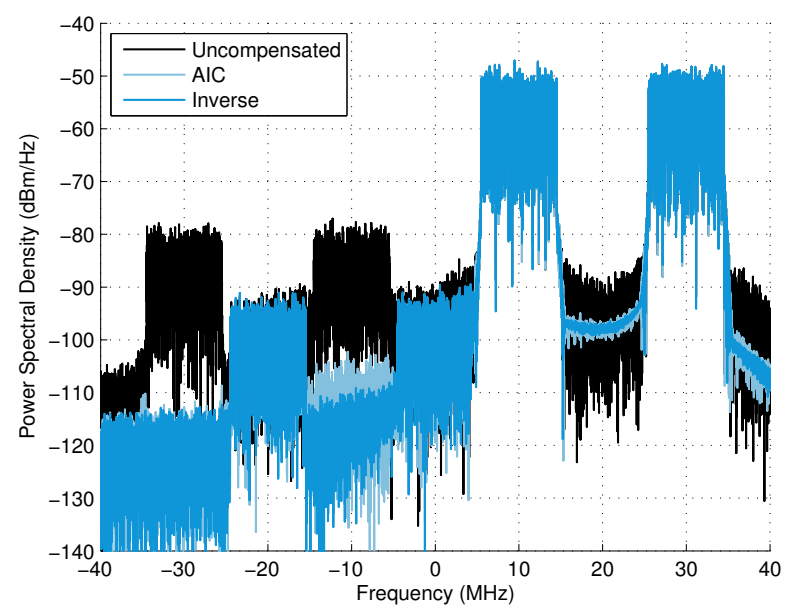

Fig. 5. Spectral illustration of nonlinearity compensation performance with two weak signals having down-converted center frequencies $-20 \mathrm{MHz}$ and $0 \mathrm{MHz}$ concurrently with two blocker signals (at $10 \mathrm{MHz}$ and $30 \mathrm{MHz}$ ), average received signal power being $-33 \mathrm{dBm}$.

provide similar DRR values for both weak signals where as AIC gives slightly lower DRR for the weak signal around $0 \mathrm{MHz}$. This is because AIC uses bandpass filter to pick the blockers and the compensation is not perfect in the filter transition band where the weak signal is partially located.

In the provided examples, both compensation methods are performing well. However, these methods have some fundamental differences which indicates that it is application specific issue which one is more suitable. AIC is able to adapt its model coefficients continuously during the normal receiver operation and is therefore able to quickly follow the changes in nonlinearities, which may happen due to the variations in environmental conditions such as in temperature. On the other hand, AIC extracts a reference signal from the received distorted signal, which decreases the accuracy of

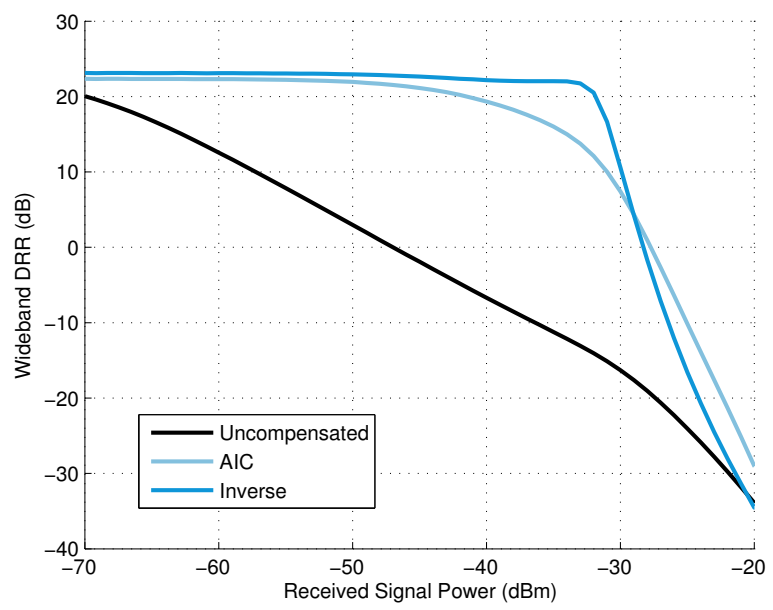

Fig. 6. Wideband distortion rejection ratio as a function of received signal power. The whole reception band excluding blockers are considered in wideband DRR.

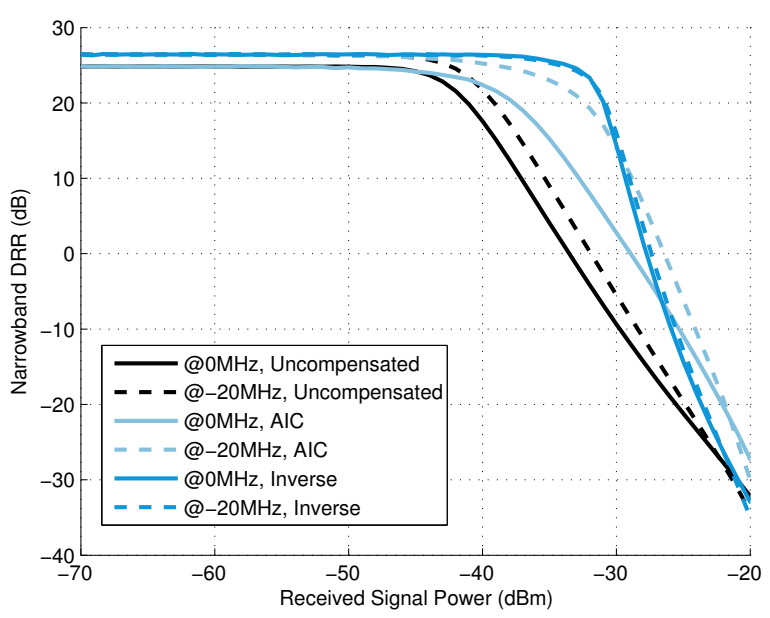

Fig. 7. Narrowband distortion rejection ratio as a function of received signal power. Narrowband DRR considers only a specific weak signal band.

the interference cancellation. The post-inverse method uses calibration signal which guarantees more accurate nonlinearity modeling. However, the calibration cannot be done during the normal receiver operation. Other difference is that the postinverse method removes distortion from the whole reception band whereas AIC is able to only cancel distortion outside the blocker bands. This may be a problem only if also the blocker signals are desired to be demodulated by the receiver. From the complexity point of view, the post-inverse method is more simple since it does not use the bandpass and bandstop filters required in AIC. Furthermore, the post-inverse method does not require the knowledge about blocker band locations and bandwidths.

It is worth noticing that the coefficients in the calibrationbased post-inverse model are independent of the received signal power, at least up to the calibration signal power. However, 
they depend on the receiver front-end nonlinearities and gains. Therefore the calibrated post-inverse model coefficients are directly valid only for certain receiver front-end configuration. If, e.g., amplifier gains are adjusted, also the optimal post-inverse model coefficients may change. One option is to calibrate different set of inverse-model coefficients for different receiver front-end configurations. Other option is to calibrate only once, but weight the inverse-model coefficients properly according to the gain adjustments. In both options, some knowledge about the receiver front-end configuration is required. This is a reasonable requirement because, e.g., the receiver gains are typically digitally controlled and therefore information about gain changes is available for the compensation method.

\section{CONCLUSION}

This paper discussed and developed digital post-processing methods for linearizing wideband radio receivers in order to enhance wideband non-contiguous spectrum sensing and access. Two methods, adaptive interference cancellation and calibration-based post-inverse, were compared with computer simulations in challenging non-contiguous spectrum access scenario with strong blocking signals. The post-inverse method is able to provide better linearization performance, but is only suitable for applications where calibration periods are allowed. Adaptive interference cancellation method is able to work without calibration, but is computationally more complex. However, both methods are waveform-independent and therefore applicable to wide variety of systems.

\section{REFERENCES}

[1] A. A. Abidi, "Direct-conversion radio transceivers for digital communications," IEEE J. Solid-State Circuits, vol. 30, no. 12, pp. 1399-1410, Dec. 1995.

[2] B. Razavi, "Design considerations for direct-conversion receivers," IEEE Trans. Circuits Syst. II, vol. 44, no. 6, pp. 428-435, June 1997.

[3] D. H. Mahrof, E. A. M. Klumperink, J. C. Haartsen, and B. Nauta, "On the effect of spectral location of interferers on linearity requirements for wideband cognitive radio receivers," in Proc. IEEE Symp. on New Frontiers in Dynamic Spectrum (DySPAN2010), Singapore, Apr. 2010 pp. 1-9.

[4] B. Razavi, "Cognitive radio design challenges and techniques," IEEE J. Solid-State Circuits, vol. 45, no. 8, pp. 1542-1553, Aug. 2010.

[5] M. Vääräkangas, S. Kallioinen, A. Pärssinen, V. Turunen, and J. Ryynänen, "Trade-offs in primary detection using a mobile phone as a sensing device," in Proc. 6th Int. ICST Conf. on Cognitive Radio Oriented Wireless Networks and Communications (CROWNCOM2011), Osaka, Japan, June 2011, pp. 241-245.

[6] M. Allén, J. Marttila, M. Valkama, S. Mäkinen, M. Kosunen, and J. Ryynänen, "Digital linearization of direct-conversion spectrum sensing receiver," in Proc. 1st IEEE Global Conf. Signal and Information Process. (GlobalSIP2013), Austin, TX, USA, Dec. 2013, pp. 1158-1161.

[7] D. Gurney, G. Buchwald, L. Ecklund, S. Kuffner, and J. Grosspietsch, "Geo-location database techniques for incumbent protection in the TV white space," in Proc. 3rd IEEE Symp. on New Frontiers in Dynamic Spectrum Access Networks (DySPAN2008), Oct. 2008, pp. 1-9.

[8] R. Murty, R. Chandra, T. Moscibroda, and P. Bahl, "SenseLess: A database-driven white spaces network," IEEE Trans. Mobile Comput., vol. 11, no. 2, pp. 189-203, Feb. 2012.

[9] Digital cellular telecommunications system (Phase 2+); Radio transmission and reception, 3GPP TS 45.005 version 11.4.0 Release 11, Jan. 2014.

[10] Universal Mobile Telecommunications System (UMTS); Base Station $(B S)$ radio transmission and reception (FDD), 3GPP TS 25.104 version 11.8.0 Release 11, Jan. 2014.
[11] LTE; Evolved Universal Terrestrial Radio Access (E-UTRA); Base Station $(B S)$ radio transmission and reception, 3GPP TS 36.104 version 11.7.0 Release 11, Jan. 2014.

[12] H. F. Lundin, "Characterization and correction of analog-todigital converters," Ph.D. dissertation, School Electrical Eng., Royal Inst.Technology, Stockholm, Sweden, 2005.

[13] J. Tsimbinos, "Identification and compensation of nonlinear distortion," Ph.D. dissertation, School Electronic Eng., Univ. of South Australia, Adelaide, Australia, 1995.

[14] J. Tsimbinos and K. V. Lever, "Nonlinear system compensation based on orthogonal polynomial inverses," IEEE Transactions on Circuits and Systems: Fundamental Theory and Applications, vol. 48, no. 4, pp. 406417, Apr 2001.

[15] M. Grimm, M. Allén, J. Marttila, M. Valkama, and R. Thomä, "Joint mitigation of nonlinear RF and baseband distortions in wideband directconversion receivers," IEEE Trans. Microw. Theory Tech., vol. 62, no. 1 pp. 166-182, Jan. 2014.

[16] M. Valkama, A. Shahed hagh ghadam, L. Anttila, and M. Renfors, "Advanced digital signal processing techniques for compensation of nonlinear distortion in wideband multicarrier radio receivers," IEEE Trans. Microw. Theory Tech., vol. 54, no. 6, pp. 2356-2366, June 2006.

[17] E. Rebeiz, A. Shahed hagh ghadam, M. Valkama, and D. Cabric, "Suppressing RF front-end nonlinearities in wideband spectrum sensing," in Proc. 8th Int. Conf. Cognitive Radio Oriented Wireless Networks (CROWNCOM2013), Washington D.C., July 2013, pp. 87-92. 